Enfermagem Brasil 2018;17(3):273-8

\title{
ARTIGO ORIGINAL \\ Validade da Escala Hospitalar de Ansiedade e Depressão no período pré-operatório de cirurgia cardíaca
}

Eduardo Tavares Gomes, M.Sc. ${ }^{*}$, Simone Maria Muniz da Silva Bezerrra, D.Sc. **

*Enfermeiro, Doutorando em Ciências pelo Programa de Pós-graduação em Enfermagem em Saúde do Adulto da Escola de Enfermagem da Universidade de São Paulo, **Enfermeira, PósDoutora em Enfermagem Fundamental pela Escola de Enfermagem de Ribeirão Preto da Universidade de São Paulo, Professora do Programa Associado de Pós-graduação em Enfermagem UPE/UEPB

Recebido em 23 de julho de 2017; aceito em 19 de março de 2018.

Endereço para correspondência: Eduardo Tavares Gomes, Hospital das Clínicas da Universidade de Federal Pernambuco, Avenida Profo Moraes Rego, 1235 Cidade Universitária 50670-901 Recife PE, E-mail: edutgs@hotmail.com; Simone Maria Muniz da Silva Bezerra: simonemunizm2@gmail.com

\section{Resumo}

Objetivo: Avaliar as propriedades psicométricas da Escala Hospitalar de Ansiedade e Depressão aplicada a pacientes internados no período pré-operatório de cirurgia cardíaca. Métodos: Trata-se de um estudo metodológico realizado entre os meses de janeiro e abril de 2017, nas enfermarias de um hospital universitário especializado em cardiologia, referência regional em cirurgia cardíaca. A escala foi aplicada a 176 pacientes internados em período préoperatório de cirurgia cardíaca de revascularização miocárdica, troca ou plastia valvar. Os dados foram analisados com recursos de estatística descritiva e inferencial, utilizando o software Epi-info 7.0 e SPSS 24.0. Resultados: A confiabilidade da aplicação na amostra foi considerada alta, tanto para a subescala Ansiedade (alfa de Cronbach $=0,815$ ), quanto para Depressão (alfa de Cronbach $=0,845$ ), indicando sua boa representação da informação desejada. Nenhuma correlação forte foi observada entre os itens ímpares, referentes à depressão, e aos pares, referentes à ansiedade. Conclusão: A Escala Hospitalar de Ansiedade e Depressão, em seus dois domínios, em todos os itens, apresentou-se válida a ser utilizada para pacientes no período pré-operatório de cirurgia cardíaca.

Palavras-chave: ansiedade, depressão, período pré-operatório, cirurgia cardíaca.

\begin{abstract}
Validity of the Hospital Anxiety and Depression Scale in the preoperative period of cardiac surgery

Objective: To evaluate the psychometric properties of the Hospital Anxiety and Depression Scale applied to hospitalized patients in the preoperative period of cardiac surgery. Methods: This is a methodological study developed between January and April 2017, in the wards of a university hospital specialized in cardiology, a regional reference in cardiac surgery. The scale was applied to 176 patients in the preoperative period of myocardial revascularization or valve surgery. Data were analyzed using descriptive and inferential statistics using Epi-info 7.0 and SPSS 24.0 software. Results: The reliability of the application in the sample was considered high, both for the subscale Anxiety (Cronbach's alpha $=0.815$ ) and for Depression (Cronbach's alpha $=0.845)$, indicating its good representation of the desired information. No strong correlation was observed between the odd items, referring to depression, and the pairs, referring to anxiety. Conclusion: The Hospital Anxiety and Depression Scale, in its two domains, in all items, was valid for use in patients in the preoperative period of cardiac surgery.

Key-words: anxiety, depression, preoperative period, cardiac surgery.

\section{Resumen}

Validez de la Escala Hospitalaria de Ansiedad y Depresión en el período preoperatorio de cirugía cardiaca

Objetivo: Evaluar las propiedades psicométricas de la Escala de Ansiedad y Depresión Hospitalaria aplicada a pacientes hospitalizados en el período preoperatorio de cirugía cardíaca.
\end{abstract}


Métodos: Se trata de un estudio metodológico desarrollado entre enero y abril de 2017, en las salas de un hospital universitario especializado en cardiología, referente regional en cirugía cardíaca. La escala se aplicó a 176 pacientes en el período preoperatorio de revascularización miocárdica o cirugía valvular. Los datos se analizaron mediante estadísticas descriptivas e inferenciales utilizando el software Epi-info 7.0 y SPSS 24.0. Resultados: La fiabilidad de la aplicación en la muestra se consideró alta, tanto para la subescala Ansiedad (alfa de Cronbach $=0,815$ ) como para la depresión (alfa de Cronbach $=0,845$ ), lo que indica una buena representación de la información deseada. No se observó una correlación fuerte entre los elementos impares, que se refieren a la depresión, y los pares, en referencia a la ansiedad. Conclusión: La Escala de Ansiedad y Depresión del Hospital, en sus dos dominios, en todos los ítems, fue válida para el uso en pacientes en el período preoperatorio de cirugía cardíaca.

Palabras-clave: ansiedad, depresión, período preoperatorio, cirugía cardíaca.

Introdução

A proximidade da cirurgia cardíaca tem como repercussão significativa a ansiedade e depressão, envolvendo sentimentos e significados diversos para os pacientes [1,2]. Os profissionais de saúde devem reconhecer a ocorrência de indicativos para estas alterações de humor, bem como sua implicação no processo de enfrentamento do trâmite cirúrgico [3,4].

Ao longo dos anos, diversas escalas para rastreamento de sinais e sintomas ansiosos foram desenvolvidas. Contudo, sintomas somáticos considerados foram sendo reconhecidos como possíveis vieses de confusão quando se aplicavam tais instrumentos a pacientes com comorbidades clínicas. Neste contexto, foi elaborada a Escala Hospitalar de Ansiedade e Depressão (HADS), que tem sido utilizada por seu uso rápido e simples (em até dez minutos), por ter sido demonstrada sua validade e confiabilidade em vários estudos e por não conter avaliação de sintomas somáticos [5].

A HADS é composta por 14 questões, sendo sete para avaliar ansiedade e sete para depressão, cada item é pontuado numa escala de 0 a 3 , num total de 21 pontos para cada escala, com ponto de corte de: sem ansiedade ou depressão de 0 a 8 , com ansiedade ou depressão $>9$ em cada subescala, respectivamente $[5,6]$.

Considerando que esta escala foi traduzida há mais de 20 anos e ainda não foi validada em suas propriedades psicométricas para a população específica de pacientes em período pré-operatório de cirurgia cardíaca, o presente estudo se destinou a avaliar as propriedades psicométricas da Escala Hospitalar de Ansiedade e Depressão aplicada a este grupo.

Material e métodos

Trata-se de um estudo metodológico para investigar propriedades psicométricas da Escala Hospitalar de Ansiedade e Depressão. O estudo foi realizado entre os meses de janeiro e abril de 2017, nas enfermarias de um hospital universitário especializado em cardiologia, referência regional em cirurgia cardíaca.

Para delimitação da amostra foi realizado um cálculo através da equação de cálculo do tamanho amostral para médias, considerando que a variável-desfecho é quantitativa contínua. Utilizou-se como referência uma publicação internacional que utilizou da versão em inglês da escala para uma população similar, visto que a HADS, em sua validação para o português no Brasil e nos artigos com pacientes dessa população, não teve divulgado o valor do desviopadrão encontrado [5-7].

Para o cálculo, utilizou-se um erro $\alpha$ de $5 \%$, que corresponde à diferença entre o valor estimado pela pesquisa e o verdadeiro valor; um nível de confiança de $95 \%$, que é a probabilidade de que o erro amostral efetivo seja menor do que o erro amostral admitido pela pesquisa. O desvio-padrão adotado com referência foi encontrado em um estudo internacional que avaliou a ansiedade e a depressão, utilizando-se da mesma escala, em 142 pacientes que se submeteram a cirurgia cardíaca, dois dias antes do evento. $O$ valor do desvio-padrão encontrado no referido estudo foi de 8,72 [7]. O erro máximo adotado foi de 1,5 ponto na média. Considerando a população finita de 200 pacientes submetidos à cirurgia cardíaca, em média, para um período de cinco meses de coleta, a amostra foi estimada em 130 pacientes. Foi determinada uma meta de coletar, no entanto, 30\% (169 pacientes) a mais, considerando as possíveis perdas, sendo, ao final, coletados um total de 176 pacientes. 
Os dados foram coletados utilizando-se um instrumento próprio contendo, entre outros: um questionário elaborado para levantamento sociodemográfico, como sexo, idade, procedência, renda, escolaridade, afiliação religiosa, tipo de cirurgia, tempo de internamento, etc.; e também a Escala Hospitalar de Ansiedade e Depressão.

Foram incluídos pacientes internados em período pré-operatório de cirurgia cardíaca de revascularização miocárdica, troca ou plastia valvar e excluídos os que apresentassem nível de consciência rebaixado, comunicação verbal prejudicada ou qualquer condição clínica ou psicológica que prejudicasse a entrevista ou a tornasse desconfortável; uso de antidepressivo; diagnóstico médico prévio referido de transtornos de humor, de ansiedade ou qualquer outro transtorno psiquiátrico.

Para cada paciente, foi aplicado o instrumento de coleta de dados descrito anteriormente, mediante entrevista. Foram realizadas 15 entrevistas piloto, de forma a familiarizar o entrevistador com o uso da escala. A coleta de dados aconteceu em duas fases principais. Na primeira etapa os participantes do estudo foram informados quanto aos procedimentos e objetivos da pesquisa e convidados à participação mediante a assinatura do Termo de Consentimento Livre e Esclarecido (TCLE). Na segunda etapa foram realizadas as entrevistas e aplicado o instrumento de coleta.

As entrevistas foram realizadas à beira-do-leito, com ciência do enfermeiro responsável pelo setor bem como os acompanhantes, que foram orientados a não intervir em nenhuma resposta se permanecessem ao lado dos pacientes.

O armazenamento dos dados primários deu-se através de planilhas do software Microsoft Excel 2013. Os dados foram analisados com recursos de estatística descritiva e inferencial, utilizando o software Epi-info 7.0 e SPSS 24.0. Através do teste de Normalidade Kolmogorov-Smirnov foram verificados que os desfechos avaliados apresentaram nenhuma distribuição normal $(<p=0,05)$. A associação entre as escalas e subescalas utilizadas foram avaliadas através do coeficiente de correlação de Pearson e, por fim, a consistência interna dos itens das escalas foi avaliada pelo alfa de Cronbach.

A pesquisa foi elaborada pautada nos preceitos da Resolução CNS no466/2012. O início da coleta de dados deu-se apenas após a apreciação e aprovação do Comitê de Ética em Pesquisa do Pronto-Socorro Cardiológico Universitário de Pernambuco - PROCAPE/UPE Parecer no.1.915.220, CAAE: 30622414.7.0000.5192.

Resultados e discussão

A Escala Hospitalar de Ansiedade e Depressão vem sendo traduzida e validada para vários idiomas ao longo dos anos, consequência de um movimento acadêmico de consideração dos fatores emocionais na saúde [8]. Estudos vêm mostrando que, para pacientes internados, a escala pode ser inclusive autoaplicável, com duração de dois a cinco minutos por paciente $[9,10]$.

Dentre os pacientes participantes no estudo, $49,1 \%$ eram do interior, $17,3 \%$ da capital e $33,5 \%$ da região metropolitana. A idade média observada foi de $59,16 \pm 13,86$ anos e quando a idade foi agrupada e classificada entre pacientes acima de 60 anos, notou-se que $55,7 \%$ deles pertenciam a essa classe. A distribuição do sexo foi equilibrada tendo $50,6 \%$ de homens e $49,4 \%$ de mulheres. A maioria dos pacientes $(54,6 \%)$ era casada e $45,4 \%$ solteiros ou sem companheiro.

Mais da metade da amostra foi composta por pacientes sem atividade laboral $(66,7 \%)$ com apenas um terço deles ativos no mercado de trabalho (33,3\%). A renda média observada entre eles foi de 1,31 $\pm 0,96$ salários mínimos (SM), 70,7\% declararam receber até $1 \mathrm{SM}$ e apenas $29,3 \%$ tinham renda superior a $1 \mathrm{SM}$. O número médio de filhos foi de $3,41 \pm 2,48$.

Apenas um pouco mais de um quinto da amostra $(22,5 \%)$ já havia sido submetida à cirurgia cardíaca prévia. Em média, os pacientes ficaram internados por 22,06 $\pm 11,08$ dias, $70,7 \%$ mais do que 15 dias; o período médio de pré-operatório igual a 20,95 $\pm 29,78 \mathrm{com} 56,9 \%$ dos pacientes em até 15 dias e $43,1 \%$ com período superior a 15 dias. Em 34,5\% dos casos houve cancelamento da cirurgia, durante o internamento atual. Para a grande maioria dos pacientes houve um acompanhante $(85,5 \%)$ e visita religiosa $(65,7 \%)$, porém uma visita diária não foi observada tão frequentemente $(24,9 \%)$. Apenas em $6,4 \%$ dos casos houve uma solicitação de visita religiosa.

O valor médio encontrado para a escala ansiedade foi igual a $5,68 \pm 4,79$, inferior ao ponto de corte (8 pontos) que classifica os pacientes como ansiosos (apenas $27,6 \%$ ). Para a 
depressão os valores são ainda melhores, o valor médio para a escala foi igual a 3,99 $\pm 4,40$ tendo apenas $17,8 \%$ dos pacientes depressivos.

A ansiedade foi avaliada pela subescala composta pelos sete itens ímpares da Escala Hospitalar de Ansiedade e Depressão (HAD), enquanto que a depressão foi avaliada pelos itens pares. (Tabela I) A confiabilidade da aplicação na amostra foi considerada alta, tanto para a subescala Ansiedade (alfa de Cronbach $=0,815$ ) quanto para Depressão (alfa de Cronbach $=$ 0,845 ), indicando sua boa representação da informação desejada. Nenhuma correlação forte foi observada entre os itens ímpares, nem os pares, apenas uma moderada entre HAD6 e HAD12 $(r=0,630)$.

Tabela I - Estatísticas descritivas para os itens que compõe as subescalas de ansiedade e depressão. Recife/PE, Brasil, 2017.

\begin{tabular}{llllll}
\hline \multicolumn{2}{l}{ Subescala Ansiedade } & \multicolumn{3}{c}{ Subescala Depressão } \\
\hline Item & Média & Desvio Padrão & Item & Média & Desvio Padrão \\
\hline HAD 1 & 1,06 & 0,95 & HAD 2 & 0,63 & 0,93 \\
HAD 3 & 0,90 & 1,09 & HAD 4 & 0,39 & 0,77 \\
HAD 5 & 0,91 & 1,07 & HAD 6 & 0,71 & 0,94 \\
HAD 7 & 0,85 & 1,18 & HAD 8 & 0,76 & 0,88 \\
HAD 9 & 0,70 & 0,88 & HAD 10 & 0,43 & 0,83 \\
HAD 11 & 0,95 & 1,04 & HAD 12 & 0,53 & 0,84 \\
HAD 13 & 0,31 & 0,61 & HAD 14 & 0,55 & 0,90 \\
\hline
\end{tabular}

Os itens da subescala ansiedade apresentaram maiores escores individuais que os da subescala depressão. (Tabela I) A correlação moderada ou fraca entre os itens dentro da própria subescala mostram que os itens avaliam um conteúdo independente do outro, sem sobreporem-se. (Tabela II) A possibilidade de retirada de um item mostrou-se diminuir a confiabilidade da escala, justificando a presença dos itens todos para a avaliação de ambos os construtos ansiedade e depressão. (Tabela III)

Tabela II - Matriz de correlação entre itens das subescalas Ansiedade e depressão. Recife/PE, Brasil, 2017.

\begin{tabular}{llllllll}
\hline & HAD 1 & HAD 3 & HAD 5 & HAD 7 & HAD 9 & HAD 11 & HAD 13 \\
\hline HAD 1 & 1,00 & 0,42 & 0,48 & 0,23 & 0,35 & 0,45 & 0,46 \\
HAD 3 & 0,42 & 1,00 & 0,46 & 0,39 & 0,52 & 0,41 & 0,39 \\
HAD 5 & 0,48 & 0,46 & 1,00 & 0,43 & 0,33 & 0,45 & 0,52 \\
HAD 7 & 0,23 & 0,39 & 0,43 & 1,00 & 0,33 & 0,31 & 0,34 \\
HAD 9 & 0,35 & 0,52 & 0,33 & 0,33 & 1,00 & 0,40 & 0,39 \\
HAD 11 & 0,45 & 0,41 & 0,45 & 0,31 & 0,40 & 1,00 & 0,40 \\
HAD 13 & 0,46 & 0,39 & 0,52 & 0,34 & 0,39 & 0,40 & 1,00 \\
\hline & HAD 2 & HAD 4 & HAD 6 & HAD 8 & HAD 10 & HAD 12 & HAD 14 \\
HAD 2 & 1,00 & 0,34 & 0,32 & 0,40 & 0,27 & 0,36 & 0,39 \\
HAD 4 & 0,34 & 1,00 & 0,53 & 0,34 & 0,45 & 0,55 & 0,52 \\
HAD 6 & 0,32 & 0,53 & 1,00 & 0,40 & 0,52 & 0,63 & 0,55 \\
HAD 8 & 0,40 & 0,34 & 0,40 & 1,00 & 0,42 & 0,45 & 0,36 \\
HAD 10 & 0,27 & 0,45 & 0,52 & 0,42 & 1,00 & 0,54 & 0,37 \\
HAD 12 & 0,36 & 0,55 & 0,63 & 0,45 & 0,54 & 1,00 & 0,51 \\
HAD 14 & 0,39 & 0,52 & 0,55 & 0,36 & 0,37 & 0,51 & 1,00 \\
\hline
\end{tabular}

Para a ansiedade, o iten que teve maior correlação com a subescala foi o $5(0,63)$, seguido do $7(0,62)$, que foram também os que mais contribuíram para a coerência interna. De modo contrário, o item que teve menos correlação com a subescala foi o $7(0,47)$, sendo também o que menos colaborou com a sua coerência interna. (Tabela III)

Pode-se afirmar ainda que na subescala depressão, os itens 6 e 4 foram os que mais se correlacionaram com o total, enquanto que o 6 e 12 foram os que mais contribuíram para a coerência interna da subescala. (Tabela III)

A validade divergente das subsescalas foi testada em vários estudos de validação, comprovando que cada uma se aplica a avaliar o seu construto de forma independente, contudo, uma revisão sistemática recente vem apontando que essa avaliação divergente entre 
ansiedade e depressão não é tão seguramente comprovada ou ainda não é tão clara, variando com o método estatístico utilizado e necessitando de mais estudos [8-19].

Em vários idiomas e populações, incluindo pacientes não-hospitalizados, a Escala Hospitalar de Ansiedade e Depressão vem sendo testada em todo mundo [8-19]. Contudo, ainda não havia sido testada para pacientes em período pré-operatório de cirurgia cardíaca. $\mathrm{Na}$ validação para as versões chinesa e alemã, as populações foram as que mais se aproximaram do presente estudo, ambas sendo validadas entre pacientes que apresentavam doença arterial coronariana $[18,19]$.

Um importante estudo realizado no Brasil, reavaliou a escala em uma população nãoclínica adequada, através de análise fatorial confirmatória, e sugeriu propostas de mudanças para os parâmetros diagnósticos da ansiedade ( $\geq 7$ pontos) e depressão ( $\geq 6$ pontos) [17].

Tabela III - Estatísticas dos itens de ansiedade e depressão. Recife/PE, Brasil, 2017.

\begin{tabular}{|c|c|c|c|c|c|}
\hline \multicolumn{6}{|c|}{ Estatísticas de item-total - subescala Ansiedade } \\
\hline & Média de & Variância de & Correlação de & Correlação & Alfa de \\
\hline & escala & escala & item total & múltipla ao & Cronbach \\
\hline & $\begin{array}{l}\text { se o item } \\
\text { for excluído }\end{array}$ & $\begin{array}{l}\text { se o item } \\
\text { for excluído }\end{array}$ & corrigida & quadrado & $\begin{array}{l}\text { se o item for } \\
\text { excluído }\end{array}$ \\
\hline HAD 1 & 4,63 & 17,67 & 0,55 & 0,36 & 0,79 \\
\hline HAD 3 & 4,78 & 16,33 & 0,62 & 0,41 & 0,78 \\
\hline HAD 5 & 4,78 & 16,37 & 0,63 & 0,44 & 0,78 \\
\hline HAD 7 & 4,83 & 17,02 & 0,47 & 0,26 & 0,81 \\
\hline HAD 9 & 4,98 & 18,13 & 0,54 & 0,34 & 0,79 \\
\hline HAD 11 & 4,73 & 17,04 & 0,56 & 0,33 & 0,79 \\
\hline HAD 13 & 5,37 & 19,44 & 0,59 & 0,37 & 0,79 \\
\hline \multicolumn{6}{|c|}{ Estatísticas de item-total - subescala Depressão } \\
\hline & $\begin{array}{l}\text { Média de } \\
\text { escala se o } \\
\text { item for } \\
\text { excluído }\end{array}$ & $\begin{array}{l}\text { Variância de } \\
\text { escala se o } \\
\text { item for } \\
\text { excluído }\end{array}$ & $\begin{array}{l}\text { Correlação de } \\
\text { item total } \\
\text { corrigida }\end{array}$ & $\begin{array}{l}\text { Correlação } \\
\text { múltipla ao } \\
\text { quadrado }\end{array}$ & $\begin{array}{l}\text { Alfa de } \\
\text { Cronbach } \\
\text { se o item for } \\
\text { excluído }\end{array}$ \\
\hline HAD 2 & 3,37 & 15,13 & 0,47 & 0,25 & 0,84 \\
\hline HAD 4 & 3,61 & 15,04 & 0,63 & 0,42 & 0,82 \\
\hline HAD 6 & 3,28 & 13,74 & 0,68 & 0,51 & 0,81 \\
\hline HAD 8 & 3,24 & 14,98 & 0,54 & 0,31 & 0,83 \\
\hline HAD 10 & 3,56 & 14,95 & 0,58 & 0,38 & 0,83 \\
\hline HAD 12 & 3,46 & 14,18 & 0,71 & 0,53 & 0,81 \\
\hline HAD 14 & 3,45 & 14,35 & 0,62 & 0,42 & 0,82 \\
\hline
\end{tabular}

A Escala Hospitalar de Ansiedade e Depressão apresentou boa confiabilidade para uso em entrevista junto a pacientes no período pré-operatório de cirurgia cardíaca. Os itens foram avaliados com boa relação com a avaliação do construto contemplado em cada subescala, tendo contribuições independentes uns dos outros, ou seja, justificando sua presença na escala.

Como limitação do estudo, vale ressaltar que não houve validação concorrente com outro instrumento de rastreabilidade de ansiedade e depressão, tampouco não houve validação de concordância entre juízes.

\section{Referências}

1. Gomes ET, Espinha DCM, Bezerra SMMS. Religiosidade e crença em Deus no préoperatório de cirurgia cardíaca: estudo exploratório. Online Braz J Nurs 2015;14(3):273-83.

2. Gonçalves KKN, Silva JI, Gomes ET, Pinheiro LLS, Figueiredo TR, Bezerra SMMS. Anxiety in the preoperative period of heart surgery. Rev Bras Enferm 2016;69(2):374-80. 
3. Koerich C, Baggio MA, Erdmann AL, Lanzoni GMM, Higashi GDC. Revascularização miocárdica: estratégias para o enfrentamento da doença e do processo cirúrgico. Acta Paul Enferm 2013;26(1):8-13.

4. Rodrigues HF, Furuya RK, Dantas RAS, Dessotte CAM. Ansiedade e depressão em cirurgia cardíaca: diferenças entre sexo e faixa etária. Esc Anna Nery 2016;20(3):e20160072.

5. Carneiro AF, Mathias LAST, Rassi Júnior A, Morais NS, Gozzani JL, Miranda AP. Evaluation of preoperative anxiety and depression in patients undergoing invasive cardiac procedures. Rev Bras Anestiol 2009;59(4):431-8.

6. Marcolino JAM, Mathias LAST, Piccinini Filho L, Guaratini AA, Suzuki FM, Alli LAC. Hospital Anxiety and Depression Scale: a study on the validation of the criteria and reability on preoperative patients. Rev Bras Anestesiol 2007;57(1):52-62.

7. Krannich JHA, Weyers P, Lueger S, Herzog M, Bohrer T, Elert O. Presence of depression and anxiety before and after coronary artery bypass graft surgery and their relationship to age. BMC Psychiatry 2007;7:47.

8. Snaith RP. The Hospital Anxiety and Depression Scale. Health Qual Life Outcomes 2003;1:29.

9. Bjelland I, Dahl AA, Haug TT, Neckelmann D. The validity of the Hospital Anxiety and Depression Scale; an updated review. J Psychiat Res 2002;52:69-77. doi: 10.1016/S0022-3999(01)00296-3.

10. Bunevicius A, Staniute M, Brozaitiene J, Bunevicius R. Diagnostic accuracy of selfrating scales for screening of depression in coronary artery disease patients. $J$ Psychosom Res 2012;72(1):22-5.

11. Helvik AS, Engedal K, Skancke RH, Selbæk G. A psychometric evaluation of the Hospital Anxiety and Depression Scale for the medically hospitalized elderly. Nord J Psychiatry 2011;65(5):338-44.

12. Kjaergaard M, Arfwedson WCE, Waterloo K, Jorde R. A study of the psychometric properties of the Beck Depression Inventory-II, the Montgomery and Åsberg Depression Rating Scale, and the Hospital Anxiety and Depression Scale in a sample from a healthy population. Scand J Psychol 2014;55(1):83-9.

13. Quintana JM, Padierna A, Esteban C, Arostegui I, Bilbao A, Ruiz I. Evaluation of the psychometric characteristics of the Spanish version of the Hospital Anxiety and Depression Scale. Acta Psychiatr Scand 2003;107(3):216-21.

14. Preljevic VT, Østhus TB, Sandvik L, Opjordsmoen S, Nordhus IH, Os I, Dammen T. Screening for anxiety and depression in dialysis patients: comparison of the Hospital Anxiety and Depression Scale and the Beck Depression Inventory. J Psychosom Res 2012;73(2):139-44.

15. Cosco TD, Doyle F, Ward M, McGee H. Latent structure of the Hospital Anxiety and Depression Scale: a 10-year systematic review. J Psychosom Res 2012;72(3):180-4.

16. Krespi Boothby MR, Hill J, Holcombe C, Clark L, Fisher J, Salmon P. The accuracy of HADS and GHQ-12 in detecting psychiatric morbidity in breast cancer patients. Turk Psikiyatri Derg 2010;21(1):49-59.

17. Faró A. Análise Fatorial Confirmatória e Normatização da Hospital Anxiety and Depression Scale (HADS). Psic: Teor Pesq 2015;31(3):349-53.

18. Wang W, Chair SY, Thompson DR, Twinn SF. A psychometric evaluation of the Chinese version of the Hospital Anxiety and Depression Scale in patients with coronary heart disease. J Clin Nurs 2009;18(17):2436-43.

19. Emons WHM, Sijtsma K, Pedersen SS. Dimensionality of the Hospital Anxiety and Depression Scale (HADS) in Cardiac Patients: Comparison of Mokken Scale Analysis and Factor Analysis. Assessment 2010;19(3):337-53. 